\title{
Avaliação da Força Muscular Respiratória e da Função Pulmonar em Pacientes com Insuficiência Cardíaca
}

\author{
Evaluation of Respiratory Muscle Strength and Pulmonary Function in Heart Failure Patients

\begin{abstract}
Luiz Alberto Forgiarini Junior, Angélica Rubleski, Douglas Garcia, Juliana Tieppo, Rafael Vercelino, Adriane Dal Bosco, Mariane Borba Monteiro, Alexandre Simões Dias

Centro Universitário Metodista - IPA, Irmandade Santa Casa de Misecórdia de Porto Alegre, Fundação Faculdade Federal de Ciências Médicas de Porto Alegre - Porto Alegre, RS - Brasil
\end{abstract}

\section{Resumo}

Fundamento: O termo insuficiência cardíaca (IC) refere-se à falha do coração em bombear sangue para suprir as necessidades do organismo. A função pulmonar e os músculos respiratórios podem estar afetados e os sintomas típicos apresentados pelos pacientes são desconforto aos mínimos esforços.

Objetivo: Verificar a função pulmonar e a força dos músculos respiratórios em pacientes com IC em classes funcionais II e III, segundo a New York Heart Association (NYHA).

Métodos: O estudo foi descritivo e observacional, sendo incluídos 12 indivíduos com IC em classes II e III que estavam em acompanhamento ambulatorial. A função pulmonar (volume expiratório forçado no primeiro segundo - VEF1 - e capacidade vital forçada - CVF) foi avaliada por meio da microespirometria e a força muscular respiratória (pressão expiratória máxima - PEmáx - e pressão inspiratória máxima - PImáx), por meio de manovacuometria (marca Globalmed $\left.^{\circledR}\right)$.

Resultados: Houve diferença entre as classes II e III em relação à função pulmonar (VEF1: II = 91,17 $\pm 19,87$ e III $=$ $68,17 \pm 21,78 ;$ CVF: II $=68,17 \pm 21,78$ e III $=73,67 \pm 22,94)$ e à força muscular respiratória (PImáx: II $=71,67 \pm$ 40,70 e III = 53,33 \pm 29,27; PEmáx: II = 98,83 \pm 34,56 e III = 58,33 $\pm 15,06$ ). A classe II apresentou valores maiores que a III, em todos os parâmetros avaliados, com diferença estatisticamente significativa na PEmáx.

Conclusão: A função pulmonar e a força muscular respiratória estão prejudicadas na IC, onde os indivíduos da classe III apresentam valores menores que a II, principalmente na PEmáx. (Arq Bras Cardiol 2007; 89(1) : 36-41)

Palavras-chave: Insuficiência cardíaca, baixo débito cardíaco, força muscular, função pulmonar, insuficiência respiratória.

\section{Summary}

Background: Heart failure (HF) is the inability of the heart to pump enough blood to supply the necessities of the body. Pulmonary function and respiratory muscles can be affected and typical symptoms presented by the patients include discomfort at a minimal exertion.

Objective: To verify pulmonary function and respiratory muscle strength in patients with class II and III HF as defined by the New York Heart Association (NYHA).

Methods: The study was descriptive and observational, and comprised 12 class II and III HF patients in follow-up at the out-patient. Pulmonary function assessments [Forced Expiratory Volume in the first second (FEV1) and Forced Vital Capacity (FVC)] were performed using microspirometry and respiratory muscle strength [Maximal Expiratory Pressure (MEPmax) and Maximal Inspiratory Pressure (MIPmax)] were evaluated using a pressure transducer (Globalmed ${ }^{\mathrm{TM}}$ ).

Results: Differences were found between the functional classes II and III in relation to pulmonary function: FEV1 (II: $91.17 \pm 19.87$; III:

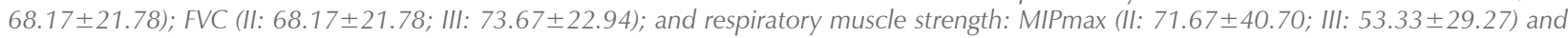

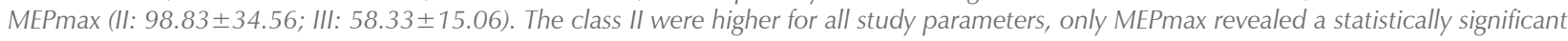
difference.

Conclusion: The pulmonary function and respiratory muscle strength are impaired in heart failure patients class III patients, particularly in relation to MEPmax. (Arq Bras Cardiol 2007; 89(1) : 32-36)

Key words: Heart failure; cardiac output, low; muscle strength, pulmonary function, respiratory insufficiency.

Full texts in English - http://www.arquivosonline.com.br

Correspondência: Alexandre Simões Dias •

Rua Hipólito da Costa, 583/18 - Morro Santa Teresa - 90840-110 - Porto Alegre, RS - Brasil

E-mail: simoesdias@terra.com.br

Artigo recebido em 31/03/06; revisado recebido em 16/01/07; aceito em 26/02/07. 


\section{Artigo Original}

\section{Introdução}

O termo insuficiência cardíaca (IC) refere-se à falha do coração em bombear sangue para suprir as necessidades de todo o organismo. Manifesta-se quando há redução do débito cardíaco ou acúmulo de sangue nas veias que chegam nos átrios direito e esquerdo (veia cava superior, veia cava inferior e veias pulmonares). Em resposta a essa situação, o sistema cardiovascular utiliza alguns mecanismos compensatórios, como elevar a freqüência cardíaca, a pressão diastólica final, bem como a massa ventricular. No entanto, se a doença continuar progredindo haverá perda da função ventricular ${ }^{1}$.

No Brasil, dados do Ministério da Saúde relatam que a IC aparece como a principal causa de internações hospitalares, com cerca de 450 mil casos novos a cada $a_{n}^{2}$. O principal sintoma apresentado pelo paciente é o cansaço aos esforços, que determina limitação para a realização das atividades da vida diária.

A função dos músculos respiratórios pode estar afetada na presença de doenças relacionadas ao coração, situação em que os pacientes podem apresentar fraqueza e falência da musculatura respiratória ${ }^{3}$.

A IC determina alterações hemodinâmicas, principalmente pela sobrecarga de pressão e de volume, na região alveolocapilar, caracterizando a fase aguda, que ainda é reversível. Assim, ocorre o remodelamento dos capilares pulmonares e da membrana tecidual, que acarreta aumento da densidade da matriz celular e prejuízo da permeabilidade endotelial. A remoção do fluido capilar ativo resulta em dificuldades nas trocas gasosas ${ }^{4}$.

Hammond e cols. ${ }^{5}$ demonstraram presença de fraqueza muscular respiratória em pacientes cardiopatas, o que reduz o fluxo sanguíneo para os músculos respiratórios, gerando atrofia muscular generalizada.

Um programa de treinamento muscular específico para musculatura respiratória melhora a força muscular, a capacidade funcional, e a qualidade de vida de pacientes com IC e fraqueza muscular inspiratória ${ }^{6}$.

Este estudo teve como objetivo avaliar a função pulmonar e a força dos músculos respiratórios em pacientes com IC em classes funcionais II e III segundo a New York Heart Association (NYHA), bem como relacionar a função pulmonar com a força muscular respiratória.

\section{Métodos}

O presente estudo teve caráter descritivo e observacional. A população do estudo foi composta por indivíduos com IC em classes II e III da NYHA, que estavam em acompanhamento no Ambulatório de Insuficiência Cardíaca do Serviço de Cardiologia da Policlínica Santa Clara do Complexo Hospitalar Santa Casa de Misericórdia de Porto Alegre, no Estado do Rio Grande do Sul. Esses pacientes apresentavam estabilidade clínica superior a três meses e estavam em acompanhamento com a equipe médica do hospital. Foram considerados critérios de exclusão: doença pulmonar avançada, tumor pulmonar, classes funcionais I e IV, e presença de alterações durante a coleta de dados, tais como dispnéia intensa, saturação de oxigênio abaixo de $80 \%$ e incompreensão, por parte dos pacientes, para a realização do teste ou pacientes que, durante o período do estudo, não participariam do mesmo.

Como instrumentos de coleta foram utilizados o manovacuômetro analógico da marca Globalmed ${ }^{\circledR}$, para avaliar a força muscular respiratória, o espirômetro da marca Sherwood Medical, modelo Respiradyne II Plus ${ }^{\circledR}$, para avaliar a função pulmonar, e a ficha de avaliação elaborada pelos pesquisadores.

Após a inclusão dos pacientes no estudo, todos os procedimentos que seriam realizados durante o trabalho foram esclarecidos por meio do termo de consentimento informado. Posteriormente foi preenchida a ficha de avaliação e foram realizados testes de força muscular e de função pulmonar.

Após a aprovação pelo Comitê de Ética e Pesquisa da Irmandade Santa Casa de Misericórdia, os dados referentes à avaliação dos músculos respiratórios foram mensurados com o paciente sentado, com os cotovelos fletidos e as mãos segurando firmemente o bocal próximo à boca. Para avaliação da pressão inspiratória máxima (PImáx), foi solicitado ao indivíduo que realizasse inspiração máxima, até o volume residual (VR), e após posicionamento adequado do equipamento na boca do paciente foi realizada expiração forçada. Para avaliar a pressão expiratória máxima (PEmáx), foi solicitado ao indivíduo que iniciasse a partir da capacidade pulmonar total (CPT), seguida pela realização de expiração forçada, observandose se o equipamento estava adequadamente posicionado na boca do paciente e adicionando-se uma presilha nasal para evitar escape aéreo ${ }^{7,8}$. O teste para avaliar a função pulmonar foi realizado por três vezes, com duração média de seis segundos cada. A coleta de dados foi feita sempre pelo mesmo avaliador, e os pacientes foram devidamente orientados sobre os procedimentos a serem realizados. Todos os testes foram realizados com os pacientes sentados, com as mãos segurando firmemente o equipamento na boca e com o nariz obstruído pela presilha apropriada. Posteriormente, solicitou-se ao paciente que realizasse inspiração até a CPT seguida de expiração forçada máxima, repetindo-se o processo por três vezes e selecionando-se a de maior valor. A diferença não poderia ultrapassar 10\% entre as repetições. Foram considerados normais os valores que ficaram acima de $80 \%$ da capacidade vital forçada (CVF). O volume expiratório forçado no primeiro segundo (VEF1) teve como referência a altura e a idade de cada indivíduo ${ }^{9}$.

A força muscular respiratória e a função pulmonar dos pacientes com IC foram avaliadas por meio das variáveis mensuradas. Foi realizada comparação entre os indivíduos da mesma classe funcional e entre as classes II e III. Para isso, foram utilizados o teste $t$ de Student, com nível de significância de 5\% ( $<<0,05$ ), e a Regressão Linear Simples, para avaliar a correlação entre as variáveis avaliadas.

\section{Resultados}

A amostra foi composta por 12 indivíduos com IC, sendo seis em classe funcional II (duas mulheres e quatro homens) e seis em classe funcional III (duas mulheres e quatro homens), conforme a classificação da NYHA, a idade variou entre 47 e 67 anos, e o peso corporal variou de $37 \mathrm{~kg}$ a $87 \mathrm{~kg}$. As características gerais da amostra estão descritas, de forma 
resumida, na tabela 1 . Todos os pacientes eram da raça branca e as doenças de base encontradas foram: cardiomiopatia (34\%), doença hipertensiva (25\%), alcoolismo (17\%), doença valvar $(8 \%)$, doença isquêmica (8\%), e doenças isquêmica e valvar associadas (8\%).

Houve diferença em relação à função pulmonar e à força muscular respiratória entre as classes funcionais II e III. Diferença estatisticamente significativa foi encontrada apenas na PEmáx quando se compararam as classes II e III (tab. 2).

Houve maior prejuízo da função pulmonar (tab. 3) e da força muscular respiratória nos pacientes que estavam em classe funcional III quando comparados aos pacientes em classe funcional II, mas com diferença estatisticamente significativa ( $p$ $<0,042$ ) apenas em relação à PEmáx (fig. 1).

\section{Discussão}

Santoro e cols. ${ }^{10}$ referem que a IC é uma doença cardíaca de grande incidência em nosso meio, sendo normalmente definida como estado fisiopatológico em que há anormalidade da função cardíaca (falha do coração em bombear sangue

\begin{tabular}{|c|c|c|c|}
\hline & Classe II & Classe III & $\mathbf{p}$ \\
\hline Idade (anos) & $58,2 \pm 8,8$ & $65,2 \pm 11,3$ & 0,59 \\
\hline Peso (kg) & $63,1 \pm 12,9$ & $64,1 \pm 18,4$ & 0,45 \\
\hline Altura (cm) & $167,7 \pm 12,8$ & $164,2 \pm 10,3$ & 0,64 \\
\hline Sexo & $4 \mathrm{M} / 2 \mathrm{~F}$ & $4 \mathrm{M} / 2 \mathrm{~F}$ & - \\
\hline
\end{tabular}

suficiente para o metabolismo tecidual). A fração de ejeção cardíaca diminui na IC, contribuindo com a taxa de mortalidade de $30 \%$ por ano, podendo chegar a $50 \%$ no mesmo período ${ }^{11}$.

Na fase avançada da IC, sinais e sintomas como dispnéia, taquipnéia, ortopnéia, extremidades frias, pálidas e cianóticas, ganho de peso, hepatomegalia, ausculta pulmonar com ruídos adventícios (crepitantes) e decréscimo da tolerância aos exercícios ou às atividades da vida diária apresentam-se de forma mais exuberante, estando presentes também na manifestação clínica da doença ${ }^{12}$.

A apnéia do sono é um achado muito comum, atingindo cerca de $40 \%$ dos pacientes graves. Seus principais sintomas são sonolência e fadiga diurna. Quando não é diagnosticada e tratada a tempo, a apnéia do sono pode causar óbito precoce $^{13}$.

Neste estudo, a análise dos resultados constatou decréscimo da função pulmonar e da força muscular respiratória nos pacientes em classe funcional III em relação aos pacientes em classe funcional II (tab. 2), com significância estatística na comparação da PEmáx entre as classes II e III (fig. 1).

Os pacientes com comprometimento respiratório apresentaram disfunção muscular, o que contribui para intolerância aos exercícios, dispnéia e hipercapnia. Os músculos respiratórios podem melhorar sua função em resposta ao treinamento físico adequado. Os sintomas podem ser prevenidos ou reduzidos com um esquema de treinamento muscular respiratório efetivo. Após o conhecimento do funcionamento dos músculos respiratórios e dos membros superiores e inferiores, os fisioterapeutas podem desenvolver o programa de treinamento mais adequado e efetivo para os pacientes ${ }^{14}$. Esse treinamento muscular pode ser realizado

\begin{tabular}{|c|c|c|c|c|}
\hline & CVF (\%) & VEF1 (\%) & PImáx $\left(\mathrm{cmH}_{2} \mathrm{O}\right)$ & PEmáx $\left(\mathrm{cmH}_{2} \mathrm{O}\right)$ \\
\hline II & $91,17 \pm 19,87$ & $87,00 \pm 11,70$ & $71,67 \pm 40,70$ & $98,83 \pm 34,56^{*}$ \\
\hline III & $68,17 \pm 21,78$ & $73,67 \pm 22,94$ & $53,33 \pm 29,27$ & $58,33 \pm 15,06$ \\
\hline
\end{tabular}

\begin{tabular}{|c|c|c|c|c|}
\hline \multirow[b]{3}{*}{ Paciente } & \multicolumn{4}{|c|}{ Tabela 3 - Dados sobre a função pulmonar da pulação estudada } \\
\hline & \multicolumn{2}{|c|}{ Classe II } & \multicolumn{2}{|c|}{ Classe III } \\
\hline & VEF1 (\%) & CVF (\%) & VEF1 (\%) & CVF (\%) \\
\hline 1 & 75,1 & 48 & 40,2 & 75,1 \\
\hline 2 & 69,5 & 51,4 & 70,9 & 69,5 \\
\hline 3 & 95,1 & 55,6 & 68,8 & 95,1 \\
\hline 4 & 86,9 & 68,2 & 73,7 & 86,9 \\
\hline 5 & 96,7 & 101,2 & 87,3 & 96,7 \\
\hline 6 & 98,6 & 89 & 102,6 & 98,6 \\
\hline Média \pm DP & $87,00 \pm 11,70$ & $91,17 \pm 19,87$ & $73,67 \pm 22,94$ & $68,17 \pm 21,78$ \\
\hline
\end{tabular}

VEF1 - volume expiratório forçado no primeiro segundo; CVF - capacidade vital forçada; DP - desvio padrão. 


\section{Artigo Original}

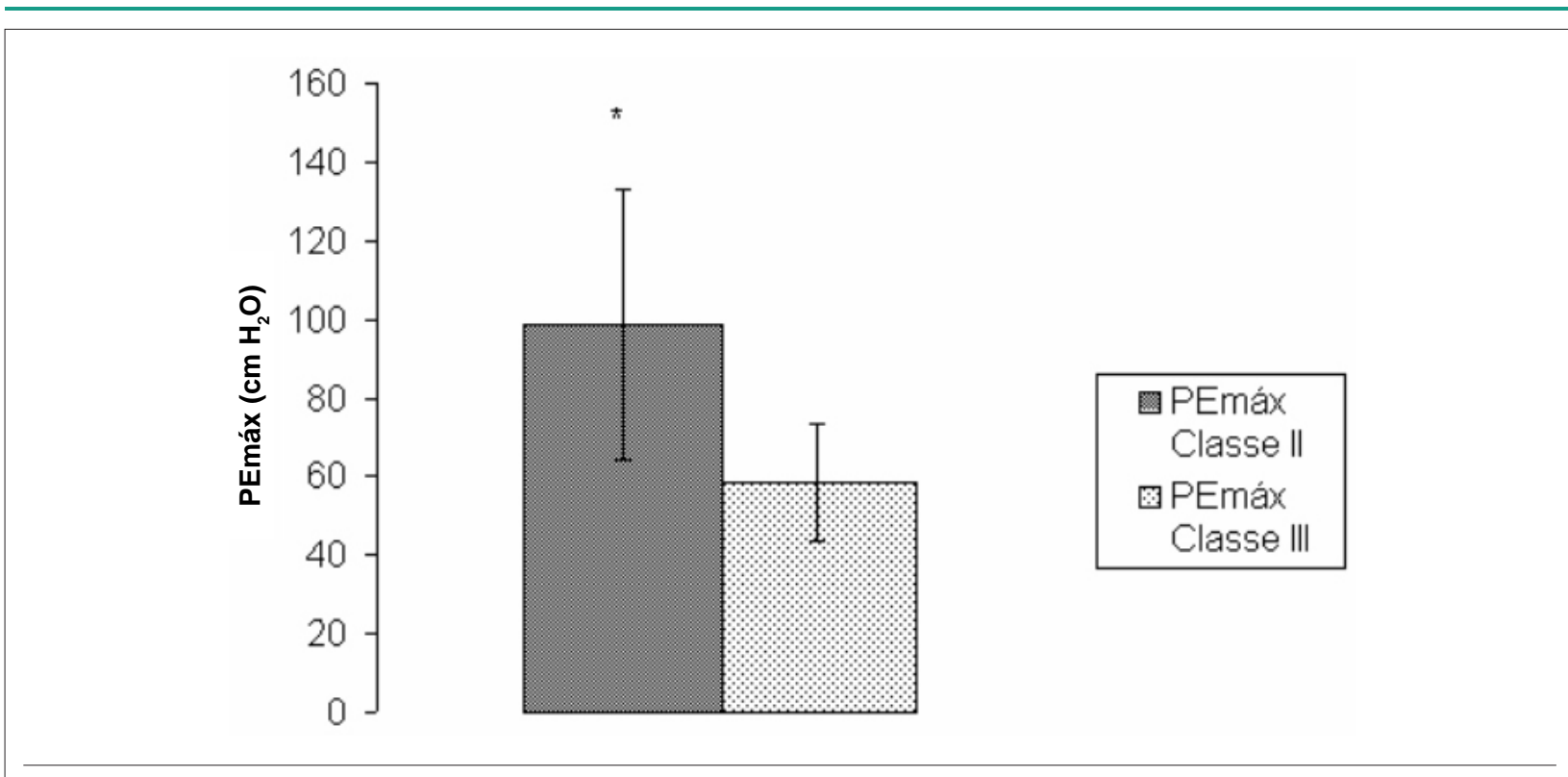

Fig. 1 - * $p=0,042$ Classe III versus Classe II.

por meio de exercícios respiratórios, por meio de exercícios aeróbicos ou com o uso de incentivadores específicos ${ }^{15}$.

Os sistemas cardiovascular e pulmonar são essenciais para a função respiratória normal, sendo responsáveis pela distribuição de oxigênio oriundo da atmosfera até os músculos esqueléticos. Como a falência do sistema cardiopulmonar causa diminuição da função pulmonar e, principalmente, da força muscular respiratória, no presente estudo a PEmáx mostrou-se menor nos pacientes mais graves ${ }^{16}$.

A força muscular respiratória alterada em pacientes com IC demonstra que existe diminuição da função pulmonar, em conseqüência da redução da força muscular respiratória. Isso foi demonstrado por Hughes e cols. ${ }^{17}$ após a avaliação dos músculos respiratórios, em que houve diminuição de $28 \%$ da PImáx e de $20 \%$ da PEmáx.

Em estudo realizado com pacientes portadores de IC em todas as classes funcionais (I, II, III e IV) da NYHA, foi observada diminuição significativa dos volumes pulmonares, especialmente na CVF e no VEF1 ${ }^{18}$. Na presente série, também foi observada diminuição da CVF e do VEF1, porém sem diferença estatisticamente significativa.

Borst e cols. ${ }^{19}$ analisaram a disfunção da musculatura respiratória em pacientes com IC, e os pacientes em classe III apresentaram prejuízo da força muscular respiratória (PImáx e PEmáx) quando comparados aos pacientes em classe II. No estudo de Borst e cols. ${ }^{19}$ foi observada maior diferença da PImáx quando comparada à PEmáx ${ }^{19}$. No presente trabalho, foi observada maior diferença na PEmáx (tab. 2; fig. 1). Segundo Meyer e cols. ${ }^{20}$, a diminuição da força muscular respiratória e da função pulmonar pode ser conseqüência da redução da massa muscular. Esse fato pode acarretar também a densidade dos capilares e a atividade das enzimas oxidativas ${ }^{20}$, que poderia ser o principal fator responsável pela atrofia do diafragma.

Evans e cols. ${ }^{21}$ encontraram diminuição da PImáx e da PEmáx de pacientes com IC, sendo mais acentuada na PImáx. O estudo demonstra a correlação entre a PImáx e os índices cardíacos, sugerindo que a perfusão muscular está envolvida na etiologia das doenças relacionadas aos músculos respiratórios ${ }^{21}$.

A inabilidade ao exercício aos mínimos esforços e o processo fisiopatológico associados à IC afetam consideravelmente os músculos esqueléticos. A musculatura esquelética dos pacientes com IC apresenta decréscimo do diâmetro das fibras dos tipos I e II. Embora nas fibras do tipo II ocorra atrofia e nas do tipo I ocorram miopatias idiopáticas e hipotrofia, a etiologia dessas mudanças primárias (miopatias musculares cardíacas ou esqueléticas) ou secundárias (relacionadas à IC) ainda não está clara. As mudanças sugerem que a fadiga muscular desses pacientes pode estar ligada à depleção da fosfocreatina a acidose intracelular, que determina o decréscimo da força muscular em até $50 \%{ }^{5}$.

Corrà e cols. ${ }^{22}$ realizaram estudo com pacientes portadores de IC para avaliar a função pulmonar e outras variáveis, cuja amostra compreendeu 323 pacientes em classes II e III da NYHA. Os autores buscaram entender qual a relação dos padrões ventilatórios com a IC. O teste consistiu de avaliação em bicicleta ergométrica, em que os pacientes foram submetidos a esforço máximo. Nesse estudo foram levados em consideração o pico de consumo de oxigênio e o padrão ventilatório. Esses autores observaram que muitos dos pacientes que apresentavam dispnéia tiveram o teste interrompido, o que evidencia diminuição da resistência física nesses pacientes. O trabalho teve duração de 11 meses. Dos 323 indivíduos incluídos no estudo, 53 morreram por apresentar pico de consumo de oxigênio menor $(42 \%$ do predito) e padrão ventilatório anormal ${ }^{22}$. Pode-se, portanto, concluir que a IC afeta também outras variáveis do sistema pulmonar, bem como o ritmo respiratório.

Johnson e cols. ${ }^{23}$, também pensando na alteração da função pulmonar durante o esforço físico, realizaram trabalho com 11 pacientes (IC classes II e III) submetidos a 
prova em esteira ergométrica. Após o término do teste foi realizada espirometria, tendo sido observada diminuição da CVF e do VEF1 dos pacientes quando comparados ao grupo controle. Esses mesmos pacientes atingiram média de $76 \%$ do predito para CVF e de $78 \%$ do predito para VEF1, enquanto o grupo controle apresentou valores de $99 \%$ e $103 \%$, respectivamente ${ }^{23}$.

Nanas e cols. ${ }^{8}$ conseguiram explicar, em parte, qual a relação do desempenho muscular respiratório com a fadiga em pacientes portadores de IC. Esses autores estudaram 55 pacientes em classes funcionais I a III da NYHA, submetendo os mesmos a prova em esteira por 15 minutos. Foram avaliados a força muscular respiratória, por meio da PImáx e da PEmáx, a função pulmonar, por meio da espirometria, e o pico de consumo de oxigênio $\left(\mathrm{VO}_{2}\right)$, com os pacientes primeiramente em repouso e após o teste (dois, cinco e 10 minutos). Nenhum paciente apresentou alteração da função pulmonar, pois todos apresentaram CVF e VEF1 acima de 80\% do predito. É interessante observar que 11 pacientes apresentaram PImáx diminuída em repouso após o teste. Assim como no presente estudo, esses autores também observaram diminuição da PImáx e da PEmáx em repouso nos paciente que apresentaram fraqueza muscular antes do teste. A média de PImáx ficou em $73 \%$ do predito, enquanto a da PEmáx ficou em 53\% do predito. Observando os dados expostos nesse estudo, pode-se dizer que a musculatura expiratória estava prejudicada no final do teste, já que seus valores médios caíram para $49 \%$ do predito, enquanto os valores da PImáx caíram para $72 \%$ do predito.

Coirault e cols. ${ }^{24}$, a partir do conhecimento de que a IC provoca queda do rendimento da musculatura respiratória, principalmente de sua força, iniciaram tratamento com um medicamento da classe dos inibidores de conversão, com o objetivo de aumentar a força muscular respiratória. Para esse estudo foram selecionados 18 pacientes em classes funcionais I, II e III da NYHA, os quais receberam inibidor da enzima de conversão da angiotensina durante seis meses. Antes do tratamento, a PImáx e a PEmáx apresentavam-se diminuídas

\section{Referências}

1. Aires MM. Fisiologia. 2a ed. Rio de Janeiro: Guanabara Koogan; 1999. p. 502-3.

2. Nastari L, Mady C. Tratamento da insuficiência cardíaca. Rev Bras Cardiol. 2000; 2 (5):165-73.

3. Azeredo C. Fisioterapia respiratória moderna. 3a ed. São Paulo: Manole; 1993.

4. Guazzi M. Alveolocapilar membrane dysfunction in heart failure. Chest. 2003; 124: 1090-2

5. Hammond MD, Bauer KA, Sharp JT. Respiratory muscle strength in congestive heart failure. Chest. 2004; 98: 1091-4.

6. Dall'Ago P, Chiappa G, Guths H, Stein R, Ribeiro J. Inspiratory muscle training with heart failure and inspiratory muscle weakness. J Am Coll Cardiol. 2006; 47: 757-63.

7. Black LF, Hyatt RE. Maximal respiratory pressures: normal values and em $57 \%$ e em $62 \%$ do predito, respectivamente ${ }^{24}$. Esses valores são próximos aos encontrados neste estudo no que se refere à PEmáx.

No presente estudo, foi observada redução de todos os parâmetros avaliados nos pacientes em classe III comparativamente aos pacientes em classe II, mas com significância estatística somente na PEmáx. Um dos fatores limitantes foi o pequeno número de pacientes incluídos no estudo. Assim, sugere-se a realização de investigações futuras com maior número de pacientes e a utilização de variáveis que possam especificar as reais condições clínicas, como hipertensão, diabete melito, cirurgias cardíacas, insuficiência renal e dados laboratoriais.

\section{Conclusão}

A partir dos resultados deste estudo, pode-se concluir que a função pulmonar e a força muscular respiratória estão prejudicadas em pacientes com IC, e que aqueles em classe funcional III apresentam diminuição significativa da PEmáx.

Os achados da pesquisa nos remetem à importância do acompanhamento fisioterapêutico de indivíduos com IC, nos quais o treinamento específico para os músculos respiratórios poderia melhorar seus sintomas e seu prognóstico.

\section{Potencial Conflito de Interesses}

Declaro não haver conflitos de interesses pertinentes.

\section{Fontes de Financiamento}

Esse estudo foi financiado com recursos próprios do investigador.

\section{Vinculação Acadêmica à Pós-Graduação}

Não há vinculação desse estudo a programas de pósgraduação. relationship to age and sex. Am Rev Resp Dis. 1969; 99: 696-702.

8. Nanas S, Nanas J, Kassiotis C, Alexopoulos G, Samakovli A, Kanakakis J, et al. Respiratory muscle performance is related to oxygen kinetics during maximal exercise and early recovery in patients with congestive heart failure. Circulation. 1999; 100 (5): 503-8.

9. Diretrizes para Teste de Função Pulmonar. J Pneumol. 2002; 28 (Sup 3): 1-81.

10. Santoro C, Cosmas A, Forman D, Morghan A, Bairos L, Levesque S, et al Exercise training alters skeletal muscle mitochondrial morphometric in heart failure patients. J Cardiovasc Risk. 2002; 9 (6): 377-81.

11. Villacorta H, Guimarães MP, Mesquita E. Clínicas de insuficiência cardíaca: tratamento e prevenção focados na educação do paciente. Rev Bras Cardiol. 2002; 2 (6): 205-13.

12. Cahalin LP. Heart failure. Phys Ther. 1996; 76: 517-31.

13. Kohnlein T, Welte T, Tan LB, Elliot MW. Central sleep apnea syndrome in 


\section{Artigo Original}

patients with chronic heart disease: a critical of the current literature. Thorax 2002; 57: 547-54

14. Reid W, Dechman G. Considerations when testing and training the respiratory muscles. Phys Ther. 1995; 75 (11): 971-83.

15. McKelvie R, Teo KK, Roberts R, McCartney N, Humen D, Montague T, et al. Effects of exercise training in patients with heart failure: The Exercise Rehabilitation Trial (EXERT). Am Heart J. 2002; 144 (1): 23-30.

16. Peel C. The cardiopulmonary system and movements dysfunction. Phys Ther 1996;76(5):449-55.

17. Hughes P, Polfey M, Harris M, Andrew J, Moxham J, Green M. Diaphagm strength in chronic heart failure. Am J Resp Crit Care Med. 1999; 160: 529-34.

18. Johnson B, Olson LJ, O'Malley KA, Allison TG, Squires RW. Pulmonary function in patients with reduced left ventricular function. Chest. 2001; 120 (6): 1869-76

19. Borst MM, Meyer FJ, Zugck C, Kirschke A, Schellberg D, Kubler W, et al.
Respiratory muscle dysfunction in congestive heart failure. Circulation. 2001; 103: $2153-8$

20. Meyer FJ, Zugck C, Haass M, Otterspoor L, Strasser RH, Kübler W, et al. Inefficient ventilation and reduced respiratory muscle capacity in congestive heart failure. Basic Res Cardiol. 2002; 95: 333-42.

21. Evans SA, Watson L, Hawkins M, Cowley AJ, Johnston IDA, Kinnear W. Respiratory muscle strength in chronic heart failure. Thorax. 1995; 50: 625-8.

22. Corrà U, Giordano A, Bosimini E, Mezzani A, Piepoli M, Coats A, et al. Oscillatory ventilation during exercise in patients with chronic heart failure. Chest. 2002; 121: 1572-80.

23. Johnson BD, Beck KC, Olson LJ, O'Malley KA, Allison TG, Squires RW, et al. Ventilatory constraints during exercise in patients with chronic heart failure. Chest. 2000; 117 (2): 321-32.

24. Coirault C, Hagége A, Chemla D, Fratacci MD, Guerot C, Lecarpentier I. Angiotensin-converting enzyme inhibitor therapy improves respiratory muscle strength in patients with heart failure. Chest. 2001; 115: 1755-60. 